Review

\title{
Use of Organs from Hepatitis C Viremic Donors: Addressing the Needs of a Changing Waitlist and the Effect of a Public Health Crisis
}

Brett W. Sadowski, Coleman I. Smith *

MedStar Georgetown Transplant Institute, Washington, DC, USA; E-Mails: Brett.W.Sadowski@gunet.georgetown.edu; Coleman.I.Smith@gunet.georgetown.edu

* Correspondence: Coleman I. Smith; E-Mail: Coleman.I.Smith@gunet.georgetown.edu

Academic Editor: Chung-Feng Huang

Special Issue: Unmet Need in the Management of Chronic Hepatitis C

OBM Hepatology and Gastroenterology Received: May 14, 2020

2020, volume 4, issue 3

Accepted: July 13, 2020

doi:10.21926/obm.hg.2003049

Published: July 20, 2020

\begin{abstract}
The combination of broad screening initiatives and development of effective antiviral therapies have led to a revolution in the treatment of hepatitis $C$ virus and has reduced the proportion of patients with the virus who develop a need for liver transplantation in favor of other etiologies, such as alcohol-related liver disease and non-alcoholic steatohepatitis. However, the opioid epidemic and rise in injection drug use in the United States has simultaneously led to otherwise healthy hepatitis $\mathrm{C}$ viremic patients dying of overdoses. The sum total of all of these factors has large implications for the transplant community and those patients on the wait list for multiple different organs: lower numbers of people on transplant wait list have active HCV, while HCV is becoming more common in potential liver, kidney and thoracic organ donors. We will review the history of solid organ transplant from donors with hepatitis $C$ as well as to describe the current understanding of the potential use of these grafts in light of the shifting demographics on the waiting list and potential donor population.
\end{abstract}

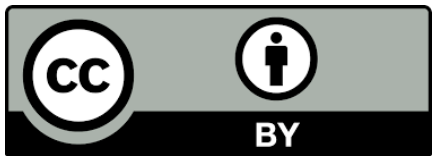

(C) 2020 by the author. This is an open access article distributed under the conditions of the Creative Commons by Attribution License, which permits unrestricted use, distribution, and reproduction in any medium or format, provided the original work is correctly cited. 


\section{Keywords}

Hepatitis C viremic donors; organs; effect of a public health crisis

\section{Introduction}

Chronic infection with hepatitis C virus (HCV) has long been one of the most common causes of chronic liver disease in the United States (US) and around the world. Cirrhosis from HCV has been a leading indication for liver transplantation (LT). With the rapid development of multiple safe, welltolerated direct acting antiviral (DAA) therapies, all genotypes of HCV can be treated with high rates of success, as opposed to previous experiences with poorly efficacious and toxic interferon-based therapies [1]. Due to this success, together with broad screening recommendations targeting at risk populations, HCV has now begun yielding its position as the top cause of registrations for LT to other etiologies such as alcohol related liver disease (ARLD) and non-alcoholic steatohepatitis (NASH) [2]. The result of these trends is that while the waitlist for LT grows thanks to advances in the management of advanced chronic liver disease, more and more people will be waiting without active HCV making expansion of the pool of potential organ donors pivotal. Simultaneously, through the combined influence of expansive opioid and injection drug use (IDU) in multiple regions in the US, a new group of potential organ donors with hepatitis $C$ has arisen. This group includes many patients much younger than those included in the birth year cohort (born between 1945 and 1965), and therefore less likely to have significant chronic liver disease themselves $[3,4]$. This narrative review will discuss the current understanding of the potential use of solid organs from HCV-viremic donors to address the burgeoning need to increase the number of donors in order to reduce wait time, reduce wait list dropout, and allow more patients access to potential life-saving therapy.

\section{Defining HCV-Positive Donors}

When discussing the role of HCV-positive organs, it is important to be precise with definitions and verbiage. The risk of HCV transmission is closely related to the presence of detectable HCV viremia. The key distinction is between donors who have detectable anti-HCV antibody without evidence of viremia (indicating either a fully treated infection, cleared infection, or less likely a false-positive antibody result) and those with detectable viral RNA via nucleic acid testing (NAT), whether they are in the acute or chronic phase [3]. HCV antibody positive donors who are NAT negative have a low risk of transmitting the virus (0-16\%), while viremic donors have a nearly $100 \%$ risk of transmission [5]. A leading consensus conference has suggested that the term HCV-viremic donor is preferred to describe a donor who has detectable viral RNA to ensure accurate communication regarding transmission risk [3].

\section{The Shifting Demographics of Donors with HCV}

The pool from which HCV-viremic donors are coming has shifted dramatically over the last few years, largely due to the opioid crisis that has swept through several regions within the United States $[3,4,6]$. 
The median age of deceased HCV seropositive donors had been in the 5 th decade for over twenty years. By 2014, the age had dropped to below 40. This serves as an indicator of the shifting of the median age of HCV infected patients away from the "baby boomer" generation (born between 1945-1965) towards persons who inject drugs (PWID) who are usually younger [7]. It also aligns with the expansion of approvals for direct acting antiviral (DAA) therapies $[8,9]$ which has successfully treated a large proportion of the "baby boomer" HCV patients. Furthermore, the increased overall all-cause mortality and comorbidity in the aging "baby boomer" population has also lowered this age. All of these factors suggest that overdose victims will continue to be the main source of viremic organ donors for years to come $[10,11]$. Another consequence of the changing epidemiology of HCV in the United States is the increase in the number of potential donors who are female which may influence size matches with smaller potential recipients, as well as add to the increased availability of organs from otherwise healthy individuals [6].

\section{Why the Hesitation in the Use of Organs from HCV Positive Donors?}

One of the major reasons for the initial reluctance to use these organs in HCV negative recipients has been founded in the fact that previous iterations of HCV treatments have been poorly tolerated and inconsistently effective. This is especially true in post-transplant infection with HCV. Furthermore, with the old interferon based regimens there was a risk of causing increased rejection of the transplanted organ. A second cause for hesitation is that even though DAA therapies are highly effective, they are not universally successful, necessitating close monitoring and backup options should first line treatments fail [12]. However, in the case of a newly transplanted solid organ this is very uncommon. Finally, we have found that fear of acquiring other donor-derived infections such as human immunodeficiency virus (HIV) which would require lifelong therapy or a bloodstream infection from a patient with endocarditis given the risks of IVDU, can also cause hesitance in patients and their providers.

As is true with many of the causes of chronic liver disease, HCV-infected recipients can have an accelerated progression of recurrent fibrosis, requiring timely follow-up prevention and treatment. Fibrosing cholestatic hepatitis ( $F C H)$ is a feared post-transplant complication of viral infection that has been more rarely seen in the current era, though should generally be considered in the right clinical setting of graft dysfunction. As is subsequently discussed, DAA therapies have altered the natural course of these complications to a far greater degree than interferon-based therapies [13, 14].

Importantly in a time when more transplants are being performed in patients with NASH, infection with hepatitis $\mathrm{C}$ in general has been associated with increased cardiovascular mortality, owing this to increased carotid atherosclerosis, insulin resistance, and ischemic heart disease [15]. Through upregulation of inflammatory markers and resultant degradation of proteins responsible for intracellular insulin signaling, chronic HCV infection has been associated with an 11-fold increased risk of incident type 2 diabetes [16]. When these associations are added to the risks of metabolic complications related to immunosuppression regimens, mainly tacrolimus or cyclosporine, the importance of timely management of post-transplant HCV become clear. Fortunately, we are in an era 
in which many of these undesired outcomes can be aborted or at least addressed in a self-limited, highly efficacious, and tolerable fashion.

In addition to the cardiovascular risks of hepatitis $C$ and of particular concern in kidney transplant recipients who fear long-term graft losses, there are also various conditions that can affect the kidneys during the course of the chronic infection including membranoproliferative glomerulonephritis and cryoglobulinemia [17]. Lymphoproliferative disorders such as non-Hodgkin lymphoma are also rare but serious complications which require significant treatments should they arise. These complications typically occur in patients with chronic infection and so timely treatment after transplant is key to prevent complications. Nevertheless, these risks likely affect the hesitance to accept an organ from a viremic donor.

\section{Game-Changing Therapy: Direct Acting Antivirals}

Interferon based therapy has typically been limited by well-described side effects including psychiatric symptoms and flu-like illness. Interferon therapy has been associated with an increased risk of rejection of the transplanted organ, due to its immunostimulatory effect. For these reasons, in addition to low efficacy, limited the impact of its use in the post-transplant setting, though it was still utilized in some cases following liver transplant. The biggest change in the last decade in the management of hepatitis $C$ has been the advent of multiple DAA therapies that have been able to reliably cure all genotypes of HCV. Now, as a result of the highly efficacious treatment regimens and broad screening protocols, HCV has begun to yield its position as the top indication for liver transplant. Since more patients without the virus will be on transplant lists, and as noted above, donors with HCV are younger than previous decades, donors are less likely to have significant other co-morbidities. DAA therapies have tremendous potential to deepen the donor pool, reduce wait list time, and save lives [1-3]. In fact, there is growing data that the era of DAA therapies has dropped the discard rate for organs substantially. Between 2005-2010, livers from donors with hepatitis C were three times more likely to be discarded, but after 2013, this relative risk of discard dropped to 1.7 [18]. These therapies have been utilized in both renal [19-21] and thoracic organ transplants [22-23], and in more recent years, larger cohorts have been examined in the liver transplant population [24-26].

In kidney transplant, after initially demonstrating evidence that transplanting organs from viremic donors into uninfected recipients was safe and post-transplant treatment of HCV was also safe and effective [27], longer follow up data in a large multi-centered cohort has shown that in the post-DAA era, not only are seronegative patients receiving more grafts from infected donors, but also that allograft function is unchanged [21].

There has also been a growing body of evidence that in both heart and lung transplants prompt treatment of HCV is safe and effective up to a year after surgery $[22,23,28]$. Bethea and colleagues demonstrated $100 \%$ patient and graft survival at a median of 10.7 months after NAT-positive heart transplants [22] while Cypel et al demonstrated that a large majority of patients receiving NAT positive lung transplants had similar early and intermediate outcomes as patients who received HCV-negative grafts [28]. Of note, in this study, a protocol which included ex-vivo lung perfusion with ultraviolet $C$ perfusate irradiation was utilized to reduce HCV RNA concentration was utilized. Some complications, 
including a case of fibrosing cholestatic hepatitis and post-treatment relapse requiring salvage therapy were noted, but overall, differences in overall outcomes amongst the cohorts were similar. In another larger report by Woolley et al, while all thoracic transplant recipients were able to successfully achieve sustained virological response after 6 months of follow up with excellent graft function, HCV-infected lung transplant recipients did have more episodes of acute cellular rejection than those who received organs from non-infected donors [23].

The use of HCV-positive (i.e. not necessarily viremic) organs for liver transplantation was first broadly reported decades ago in the pre-DAA era, though notably, only into recipients who had end-stage liver disease related to HCV themselves. This was acceptable mainly because multiple reports indicated that there was no difference in graft or patient survival in HCV positive patients who received organs from HCV-positive donors and those who did not [29-33]. In fact, Marroquin et al found that the overall patient survival was statistically better in recipients who received HCV-positive organs at two years, though this was likely impacted by this group's lower acuity of illness [29]. However, not all HCVpositive organs are suitable for transplantation, as shown by Ballarin et al who demonstrated that there was more rapid $\mathrm{HCV}$ recurrence in patients receiving organs with underlying fibrosis, a real concern in organs from donors who may have been infected for many years (mainly the "baby boomer" generation cohort) [33]. This work underscored the importance of organ selection, particularly in that donor cohort.

Large modelling and retrospective studies have supported the use of viremic donors with prompt post-transplant DAA therapy. Chatwal et al performed a modelling study which determined that HCVnegative donors who accepted any liver regardless of HCV status had an increase in life expectancy when their Model for End-Stage Liver Disease (MELD) scores were greater than 20, with highest lifeyears gained benefit seen with a MELD of 28 [33]. This was followed by the most powerful evidence to date regarding the use of HCV-viremic organs by Cotter et al, which examined both organs from serologically positive and viremic donors over a ten-year period. In addition to showing that in the postDAA era, use of HCV antibody positive organs are being used at higher rates in both positive and negative recipients, this Scientific Registry of Transplant Recipients (SRTR) based study demonstrated that the graft survival dramatically improved with DAA therapy in all recipients, regardless of HCV antibody status. Perhaps more importantly, the study also examined 87 cases of viremic donors and non-viremic recipients, and showed no significant impact on short term graft survival. The 1- and 2year graft survival outcomes were similar regardless of the donor/recipient viremic status was prior to transplant [35]. That is to say that in the era of DAA therapy, HCV negative recipients have the same graft outcome regardless on the viremic status of their donor. More recently, early experience of favorable outcomes in prospectively analyzed cohorts of patients have begun to be reported [24-26].

\section{Details Related to Treatment}

There are several practical implications to the increased utilization of organs from serologically positive (with or without HCV viremia) donors. Factors to consider include what treatment to utilize and when to start it, as well as the interaction with recipient comorbidities and immunosuppression regimens in the post-transplant setting. 
While many of the earlier DAA treatment regimens available for HCV are only able to be utilized for some genotypes, two pan-genotypic treatments exist (gleceprevir/pibrentasvir, sofosbuvir/velpatasvir). These treatments currently recommended for use in HCV-uninfected patients with grafts include either of these two therapies daily for 8 weeks with glecaprevir/pibrentasvir or 12 weeks with sofosbuvir/velpatasvir. If the viral genotype is known, alternative regimens include 12 weeks of elbasvir/grazeprevir for treatment of genotypes 1 and 4 or ledipasvir/sofosbuvir for 12 weeks for genotypes 1, 4, 5 and 6 [36]. Gleceprevir/pibrentasvir was shown to have a 98\% rate of SVR at 12 weeks in a cohort of liver and kidney transplant recipients [37] while sofosbuvir/velpatasvir had a 96\% rate of SVR for genotypes 1-4 in liver transplant recipients [38]. These are also shown in Table 1.

Table 1 Recommend treatment regimens for HCV-unifected recipients of Organs from HCVviremic donors [36].

\begin{tabular}{cl}
\hline Regimen & Duration \\
\hline Daily glecaprevir $300 \mathrm{mg} /$ pibrentasvir $120 \mathrm{mg}$ & 8 weeks \\
Daily sofobuvir $400 \mathrm{mg} /$ velpatasvir $100 \mathrm{mg}$ & 12 weeks \\
Daily elbasvir $50 \mathrm{mg} /$ grazeprevir $100 \mathrm{mg}^{\mathrm{a}}$ & 12 weeks \\
Daily ledipasvir $90 \mathrm{mg} /$ sofosbuvir $400 \mathrm{mg}^{\mathrm{b}}$ & 12 weeks \\
\hline
\end{tabular}

${ }^{\mathrm{a}}$ For genotypes 1 and 4 in patients without baseline NS5A resistance-associates substitutions

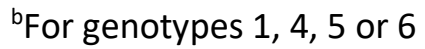

In a prospectively collected cohort of liver and kidney transplant recipients with HCV (mainly genotype 1), greater than $95 \%$ of patients treated with ledipasvir/sofosbuvir achieved SVR [39]. Kwok et al demonstrated that this regimen was highly effective in a more generalizable patient population through a multicenter retrospective study, showing high rates of SVR and only one mild case of acute rejection which was able to be treated medically [40]. Favorable post-transplant outcomes have also been seen with this regimen in the post-dual organ transplant population as well (simultaneous liver and kidney transplant) [41]. It is important to note that while sofosbuvir containing regimens were previously felt to be unsafe in patients with renal impairment, owing to increased levels of a primary metabolite known as GS-331007, the Food and Drug Administration in the US recently amended the package inserts to allow sofosbuvir containing regimens to be used in patients with kidney disease, even those on hemodialysis.

All of the treatment regimens have potential drug interactions with standard immunosuppressive regimens. Given the typical immunosuppressive regimens including calcineurin inhibitors (tacrolimus or cyclosporine), the most critical drug interaction to be aware of is with protease inhibitors (PI) component of the DAA regimen. They can significantly increase the concentration of tacrolimus and cyclosporine. With cyclosporine this increase in concentration can be several times over. The recommendation is that cyclosporine should be avoided when DAA are used. Other commonly used immunosuppressive regimens, including mTOR inhibitors (sirolimus, everolimus) or mycophenolate have less clear data, though interactions through the cytochrome p450 and P-glycoprotein have been 
reported. For more details regarding specific interaction, a useful clinical reference resource is available through the University of Liverpool at https://hep-druginteractions.org/checker, as well as www.hcvguidelines.org [36] and through resources disseminated by the American Society of Transplantation Infectious Diseases Community of Practice [42, 43].

The timing of treatment is also a factor which will play an important role moving forward in the management of previously uninfected post-transplanted patients who received organs from infected donors. There is wide variability amongst transplant centers as to when treatment should be initiated, mainly owing to the fact that payment for the treatments may not be immediately available. Recent data suggests that treatment as soon as five days after transplant is safe and highly effective at preventing HCV infection related complications [24]. Procurement for DAA therapy was obtained via a multi-step process with an institution-supported protocol, complete with a safety-net, underscoring the complexities that come when treating patients so soon after transplant when they may not have positive testing already. There is no clear data-derived answer to the question of when to initiate treatment at this point. Recommendations suggest initiation with a 'prophylactic/preemptive' strategy [34]. Additional data and experience is needed to optimize efficient clinical practices as well as interactions with payers.

\section{Proposed Protocol}

At our center, we have developed a protocol for liver and kidney transplantation for candidates without hepatitis $\mathrm{C}$ who receive organs from viremic donors. We aim in the future to add recipients of transplanted thoracic organs to our protocol. In the case of liver transplant recipients we use donor organs with minimal to no fibrosis (FO-F1). Recipients of all HCV+ organs will have negative HBsAg and a negative HIV screen. Potential recipients are counseled extensively regarding the nature purpose, potential risks, complications, benefits and alternatives to transplant with an HCV positive organ and if they are agreeable, patients are further counseled regarding potential acceptance of organs from a seropositive donor and a viremic donor. Consent is obtained during initial transplant evaluation visit and reviewed with the patient and caregivers at the time of the specific organ offer. For recipients of NAT negative organs, HCV RNA is obtained on day 1 and day 7 after transplant, then weekly for the first month, followed by monthly until HCV RNA is detected or until the 24 weeks post-transplant, indicating that no HCV infection has occurred. For recipients of NAT positive organs, HCV RNA is checked on day one after transplant and daily until it is detectable. Treatment is initiated as soon as the patient can take oral medications and the DAA therapy is available. In our experience HCV RNA becomes detectable 1-2 days after receipt of a NAT positive organ. In occasional patients HCV RNA detectability may be delayed for up to 7 days. Insurance approval is then sought. Treatment is started once approval is obtained. For most insurance payors the average time to start treatment is 30 days post-transplant. This differs somewhat from other large reports in the thoracic transplant community in which patients are started preemptively within hours of transplant [20]. We have typically used 12 weeks of a pangenotypic regimen (glecaprevir/pibrentasvir). Throughout treatment, the patients are monitored with routine lab work, tacrolimus levels, and HCV RNA levels weekly for the first month, then monthly 
through the end of treatment, as well as at weeks $4,8,12$, and 24 after the completion of treatment to ensure a sustained virologic response.

\section{Conclusion}

The shifting demographics in the liver transplant wait list as well as parallel public health crises mandate innovative practices in order to better serve the next generations of transplant candidates. Preliminary experience on the use of hepatitis $C$ viremic donor organs is promising and over the next several years, further prospective data will shed light on the long term outcomes of the patients and their grafts, we expect that the practice will become more widely utilized and the optimal treatment strategies will be determined.

\section{Author Contributions}

Both authors were involved in choosing the topic, doing the literature review as well as writing and editing the manuscript.

\section{Competing Interests}

The authors have declared that no competing interests exist.

\section{Reference}

1. Ghany MG, Marks KM, Morgan TR, Wyles DL, Aronsohn Al, Bhattacharya D, et al. Hepatitis C guidance 2019 update: AASLD-IDSA recommendations for testing, managing and treating hepatitis C virus infection. Hepatology. 2019; 71: 686-721. doi:10.1002/hep.31060.

2. Organ Procurement and Transplantation Network. Richmond: U.S Department of Health \& Human Services; 2019 [cited date 2019 December 15] Available form: https://optn.transplant.hrsa.gov/data/view-data-reports/national-data/.

3. Levitsky J, Formica RN, Bloom RD, Charlton M, Curry M, Friedewald J, et al. The American socity of transplantgation consensus conference on the use of hepatitis $C$ viremic donors in solid organ transplantation. Am J Transplant. 2017; 17: 2790-2802.

4. Gonzalez SA, Trotter JF. The rise of the opioid epidemic and hepatitis C-positive organs: A new era in liver transplantation. Hepatology. 2018; 67: 1600-1608.

5. Bari K, Luckett K, Kaiser T, Diwan T, Cuffy M, Schoech MR, et al. Hepatitis C transmission from seropositive, nonviremic donors to non-hepatitis C liver transplant recipient. Hepatology. 2018; 67: 1673-1682.

6. Rudd RA, Aleshire N, Zibbell JE, Gladden RM. Increases in drug and opioid overdose deaths: United States, 2000-2014. Morb Mortal Wkly Rep. 2016; 64: 1378-1382.

7. Suryaprasad AG, White JZ, Xu F, Eichler BA, Hamilton J, Patel A, et al. Emerging epidemic of hepatitis $C$ virus infections among young nonurban persons who inject drugs in the United States, 20062012. Clin Infec Dis. 2014; 59: 1411-1419. 
8. Manns M, Samuel D, Gane EJ, Mutimer D, McCaughan G, Buti M, et al. Ledipasvir and sofosbuvir plus ribavirin in patients with genotype 1 or 4 hepatitis $C$ virus infection and advanced liver disease: A multicenter, open-label, randomized, phase 2 trial. Lancet Infect Dis. 2016; 16: 685-697.

9. Charlton M, Everson GT, Flamm SL, Kumar P, Landis C, Brown Jr RS, et al. Ledipasvir and sofosbuvir plus ribavirin for treatment of HCV infection in patients with advanced liver disease. Gastroenterology. 2015; 149: 649-659.

10. Klevens RM, Cnary L, Huang X, Denniston MM, Yeo AE, Pesano RL, et al. The burden of hepatitis C infection-related liver fibrosis in the United States. Clin Infect Dis. 2016; 63: 1049-1055.

11. Chhatwal J, Wang X, Ayer T, Kabiri M, Chung RT, Hur C, et al. Hepatitis C disease burden in the United States in the era of oral direct-acting antivirals. Hepatology. 2016; 64: 1442-1450.

12. Chhatwal J, Chen Q, Ayer T, Bethea ED, Kanwal F, Kowdley KV, et al. Hepatitis C virus re-treatment in the era of direct acting antivirals: Projections in the USA. Aliment Pharmacol Ther. 2018; 47: 1023-1031.

13. Leroy V, Dumortier J, Coilly A, Sebagh M, Fougerou-Leurent C, Radenne S, et al. Efficacy of sofosbuvir and daclatasvir in patients with fibrosisn cholestatic hepatitis $C$ after liver transplantation. Clin Gastro Hepatol. 2015; 13: 1993-2001.

14. Forns X, Charlton M, Denning J, McHutchison JG, Symonds WT, Brainard D, et al. Sofosbuvir compassion use program for patients with severe recurrent hepatitis $C$ after liver transplantation. Hepatology. 2015; 61: 1485-1494.

15. Goossens N, Negro F. Cardiovascular manifestations of hepatitis C virus. Clin Liver Dis. 2017; 21: 465-473.

16. Hum J, Jou JH. The link between hepatitis $C$ virus and diabetes mellitus: Improvement in insulin resistance after eradication of hepatitis $C$ virus. Clin Liver Dis. 2018; 11: 73-76.

17. Cruzado JM, Gil-Vernet S, Ercilla G, Seron D, Carrera M, Bas J, et al. Hepatitis C virus-associated membranoproliferative glomerulonephritis in renal allografts. J Am Soc Nephrol. 1996; 7: 24692475.

18. Bowring MG, Kucirka LM, Massie AB, Luo X, Cameron A, Sulkowski M, et al. Changes in utilization and discard of hepatitis $C$ infected donor livers, in the recent era. Am J Transplant. 2017; 17: 519527.

19. Kucirka LM, Peters TG, Segev DL. Impact of donor hepatitis C virus infection status on death and need for liver transplant in hepatitis $C$ virus-positive kidney transplant recipients. Am J Kidney Dis. 2012; 60: 112-120.

20. Fabrizi F, Martin P, Dixit V, Messa P. Meta-analysis of observational studies: Hepatitis C and survival after renal transplant. J Viral Hepat. 2014; 21: 314-324.

21. Potluri VS, Goldberg DS, Mohan S, Bloom RD, Sawinski D, Abt PL, et al. National trends in utilization and 1- year outcomes with transplntation of HCV-viremic kidneys. J Am Soc Nephrol. 2019; 30 : 1939-1951.

22. Bethea ED, Gaj K, Gustafson JL, Axtell A, Lebeis T, Schoenike M, et al. Pre-emptive pangenotypic direct acting antiviral therapy in donor HCV-positive to recipient HCV-negative heart transplantation: An open-label study. Lancet Gastroenterol Hepatol. 2019; 4: 771-780. 
23. Woolley AE, Singh SK, Goldberg HJ, Mallidi HR, Givertz MM, Mehra MR, et al. Heart and lung transplants from HCV-infected donors to uninfected recipients. N Engl J Med. 2019; 380: 16061617.

24. Crismale JF, Khalid M, Bhansali A, De Boccardo G, Khaim R, Florman SS, et al. Liver, simultaneous liver kidney, and kidney transplantation from hepatitis C-positive donors in hepatitis C-negative recipients: A single-center study. Clin Transplant. 2020; 34: e13761.

25. Kapila N, Menon KV, Al-Khalloufi K, Vanatta JM, Murgas C, Reino D, et al. Hepatitis C virus NATpositive solid organ allografts transplanted into Hepatitis $C$ Virus-negative recipients: A real-world experience. Hepatology. 2019. doi: 10.1002/hep.31011.

26. Bethea E, Arvind A, Gustafson J, Andersson K, Pratt D, Bhan I, et al. immediate administration of antiviral therapy after transplantation of hepatitis C-infected livers into uninfected recipients: Implications for therapeutic planning. Am J Transplant. 2020; 20: 1619-1628. doi: 10.1111/ajt.15768.

27. Goldberg DS, Abt PL, Blumberg EA, Van Deerlin VM, Levine M, Reddy KR, et al. Trial of transplantation of HCV-infected kidneys into uninfected recipients. N Engl J Med. 2017; 376: 23942395.

28. Cypel M, Feld JJ, Galasso M, Ribeiro RV, Marks N, Kuczynski M, et al. Prevention of viral transmission during lung transplantation with hepatitis C-viraemic donors: An open-label, singlecentre, pilot trial. Lancet Respir Med. 2020; 8: 192-201.

29. Marroquin CE, Marino G, Kuo PC, Plotkin JS, Rustgi VK, Lu AD. Transplantation of hepatitis C positive lives in hepatitis C-positive patients is equivalent to transplanting hepatitis $\mathrm{C}$-negative livers. Liver Transpl. 2001; 7: 762-768.

30. Vargas HE, Laskus T, Wang LF, Lee R, Radkowski M, Dodson F, et al. Outcome of liver transplantation in hepatitis $\mathrm{C}$ virus-infected patients who received hepatitis $\mathrm{C}$ virus-infected grafts. Gastroenterology. 1999; 117: 149-153.

31. Saab S, Ghobrial RM, Ibrahim AB, Kunder G, Durazo F, Han S, et al. Hepatitis C positive grafts may be used in orthotopic liver transplantation: A matched analysis. Am J Transplant. 2003; 3: 11671172.

32. Stepanova M, Sayiner M, De Avila L, Younoszai Z, Racila A, Younossi ZM. Long-term outcomes of liver transplantation in patients with hepatitis $C$ infection are not affected by HCV positivity of a donor. BMC Gastroenterol. 2016; 16: 137.

33. Ballarin R, Cucchetti A, Spaggiari M, Montalti R, Di Benedetto F, Nadalin S, et al. Long-term followup and outcome of liver transplantation from anti-hepatitis $C$ virus-positive donors: $A$ european multicentric case-control study. Transplantation. 2011; 91: 1265-1272.

34. Chhatwal J, Samur S, Bethea ED, Ayer T, Kanwal F, Hur C, et al. Transplanting hepatitis C viruspositive lives into hepatitis $C$ virus-negative patients with preemptive antiviral treatment: $A$ modeling study. Hepatology. 2018; 67: 2085-2095.

35. Cotter TG, Paul S, Sandikci B, Couri T, Bodzin AS, Little EC, et al. Increasing utilization and excellent initial outcomes following liver transplant of hepatitis C virus (HCV)-viremic donors into HCVnegative recipients: Outcomes following liver transplant of HCV-viremic donors. Hepatology. 2019; 69: 2381-2395. 
36. HCV guidance: Recommendations for testing, managing, and treating hepatitis C. Danvers: The American Association for the Study of Liver Diseases and the Infectious Diseases Society of America. [cited date 2019 December 24]. Available form: https://www.hcvguidelines.org.

37. Reau N, Kwo PY, Rhee S, Brown Jr RS, Agarwal K, Angus P, et al. Glecepravir/pibrentasvir treatment in liver and kidney transplant patients with hepatitis C virus infection. Hepatology. 2018; 68: 12981307.

38. Agarwal K, Castells L, Mullhaupt B, Rosenberg WM, McNabb B, Arterburn S, et al. Sofobuvir/velpatasvir for 12 weeks in genotype 1-4 HCV infected liver transplant recipients. J Hepatol. 2018; 69: 603-607.

39. Saxena V, Khungar V, Verna EC, Levitsky J, Brown Jr RS, Hassan MA, et al. Safety and efficacy of current DAA regimens in kidney and liver transplant recipients with hepatitis $C$ : results from the HCV-TARGET study. Hepatology. 2017; 66: 1090-1101.

40. Kwok RM, Ahn J, Schiano TD, Te HS, Potosky DR, Tierney A, et al. Sofosbuvir plus ledipasvir for recurrent hepatitis $C$ in liver transplant recipients. Liver Transpl. 2016; 22: 1536-1543.

41. Nookala AU, Crismale J, Schiano T, Te H, Ahn J, Robertazzi S, et al. Direct-acting antiviral regimens are safe and effective in the treatment of hepatitis $C$ in simultaneous liver-kidney transplant recipients. Clin Transplant. 2018; 32: e13198.

42. Sparkes T, Lemonovich TL, AST Infectious Diseases Community of Practice. Interactions between anti-infective agents and immunosuppresants - Guidelines from the American Society of Transplantation Infectious Diseases Community of Practice. Clin Transplant. 2019; 33: e13510.

43. Te H, Doucette K. Viral Hepatitis: Guidelines by the American society of transplantation infectious disease community of practice. Clin Transplant. 2019; 33: e13514.

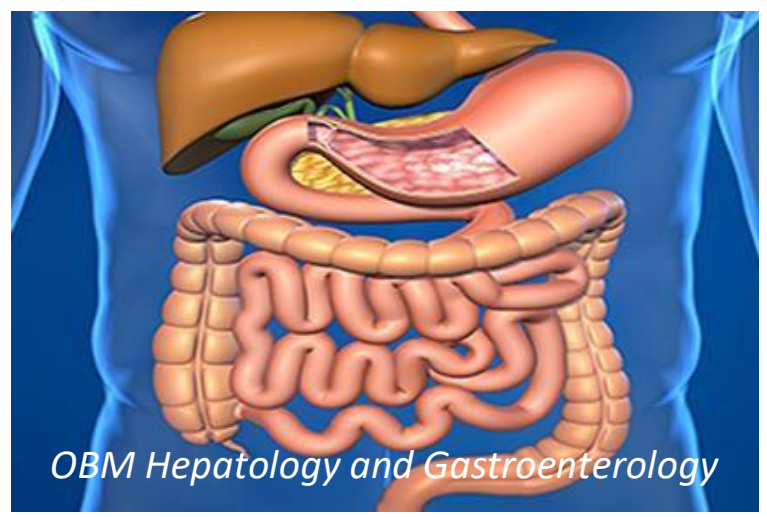

Enjoy OBM Hepatology and Gastroenterology by:

1. Submitting a manuscript

2. Joining in volunteer reviewer bank

3. Joining Editorial Board

4. Guest editing a special issue

For more details, please visit: http://www.lidsen.com/journals/hg 